\title{
Fractional Strong Matching Preclusion of Split-Star Networks ${ }^{\mathrm{a}}$
}

\author{
Ping $\mathrm{Han}^{1}$, Yuzhi Xiao ${ }^{2}$, Chengfu $\mathrm{Ye}^{1} \& \mathrm{He} \mathrm{Li}^{2}$ \\ ${ }^{1}$ School of Mathematics and Statistics, Qinghai Normal University, Xining, Qinghai 810008, China \\ ${ }^{2}$ School of Computer Science, Qinghai Normal University, Xining, Qinghai 810008, China \\ Correspondence: Ping Han, School of Mathematics and Statistics, Qinghai Normal University, Xining, Qinghai 810008, \\ China.
}

Received: May 30, 2019 Accepted: July 14, 2019 Online Published: July 17, 2019

doi:10.5539/jmr.v11n4p32ＵRL: https://doi.org/10.5539/jmr.v11n4p32

\begin{abstract}
The matching preclusion number of graph $G$ is the minimum size of edges whose deletion leaves the resulting graph without a perfect matching or an almost perfect matching. Let $F$ be an edge subset and $F^{\prime}$ be a subset of edges and vertices of a graph $G$. If $G-F$ and $G-F^{\prime}$ have no fractional matching preclusion, then $F$ is a fractional matching preclusion (FMP) set, and $F^{\prime}$ is a fractional strong matching preclusion (FSMP) set of $G$. The FMP (FSMP) number of $G$ is the minimum number of FMP (FSMP) set of $G$. In this paper, we study fractional matching preclusion number and fractional strong matching preclusion number of split-star networks. Moreover, We categorize all the optimal fractional strong matching preclusion sets of split-star networks.
\end{abstract}

Keywords: perfect matching, fractional matching preclusion number, fractional strong matching preclusion number, split-star networks

\section{AMS subject classification 2010: 05C15, 05C76, 05C78.}

\section{Introduction}

We often write $V(G)$ and $E(G)$ are vertex set and edge set, respectively. Each edge of $G$ is usually denoted by $u v$ or $v u$. If $e=u v$ is an edge of $G$, then $e$ is said to join $u$ and $v$. The minimum degree of $G$ is denoted by $\delta(G)$. A path is even path if it has even number of vertices, otherwise, is odd path. A cycle is even cycle if it has even number of vertices, otherwise, is odd cycle. A cycle (respectively, path) in $G$ that passes through each vertex of $G$ exactly once is called a Hamiltonian cycle (respectively, Hamiltonian path) of $G$. A graph that contains a Hamiltonian cycle is itself called Hamiltonian. A graph is Hamiltonian connected if there is a Hamiltonian path between every pair of vertices. Induced subgraph of graph $G$ is denoted by $G[S]$, where $S$ is nonempty subset of $V(G)$ or $E(G)$. A subgraph $H$ of a graph $G$ is called an induced subgraph if there is nonempty subset $S$ of $V(G)$ or $E(G)$ such that $H=G[S]$. The complete graph of order $n$ is denoted by $K_{n}$.

\section{1 (Strong) Matching Preclusion}

A perfect matching in a graph is a set of edges such that every vertex is incident with exactly one edge in this set. An almost perfect matching is a set of edges such that every vertex, except one, is incident with exactly one edge in this set, and the exceptional vertex is incident with none. A matching preclusion set of a graph $G$ is a set of edges whose deletion leaves the resulting graph with neither perfect matchings nor almost perfect matchings. The matching preclusion number of a graph $G$, denoted by $m p(G)$, is the size of a smallest matching preclusion set of $G$. Any such optimal set is called an optimal matching preclusion set. The concept of matching preclusion was introduced by Birgham et al. (Birgham, Harry, Biolin, \& Yellen, 2005) and further studied in (Cheng, Lesniak, Lipman, \& Lipták, 2008; Cheng, Lesniak, Lipman, Lipták, 2007; Wang, Mao, Cheng, \& Zou, 2019; Wang, Melekian, Cheng, \& Mao, 2019), with special attention to interconnection networks. For graphs with an even number of vertices, an obvious matching preclusion set is the set of edges incident with a single vertex; such a set is called a trivial matching preclusion set. A graph $G$ satisfying $m p(G)=\delta(G)$ is called maximally matched, and in a maximally matched graph some trival matching preclusion sets are optimal. If every optimal matching preclusion set is trivial, then the graph $G$ is called super matched. Being super matched is a desirable property for any real-life networks, as it is unlikely that in the event of random edge failure, every edge incident with some fixed vertex fails.

A set $F$ of edges and vertices of $G$ is a strong matching preclusion set (SMP set for short) if $G-F$ has neither perfect

\footnotetext{
${ }^{a}$ Supported by the National Science Foundation of China (Nos. 11601254, 11551001, and 61763041) and the Science Found of Qinghai Province (Nos. 2016-ZJ-948Q, 2014-ZJ-907 and 2017-ZJ-949Q).
} 


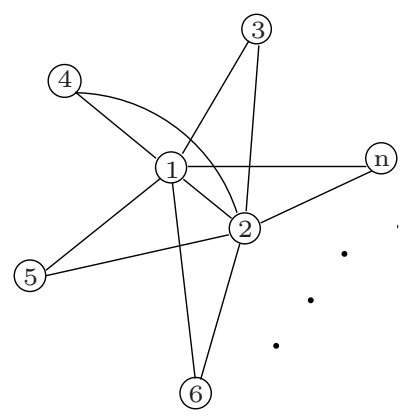

Figure 1. The generator graph for split-stars

matchings nor almost perfect matchings. The strong matching preclusion number (SMP number for short) of $G$, denoted by $\operatorname{smp}(G)$, is the minimum number of SMP sets of $G$. A SMP set is optimal if $|F|=\operatorname{smp}(G)$. The problem of strong matching preclusion set was proposed by Park and Ihm (Park \& Ihm) and further studied by (Mao, Wang, Cheng, \& Melekian, 2018), with special attention to interconnection networks. We remark that if $F$ is an optimal strong matching preclusion set, then we may assume that no edge in $F$ is incident with a vertex in $F$. It follows from the definitions of $m p(G)$ and $\operatorname{smp}(G)$ that $\operatorname{smp}(G) \leq m p(G) \leq \delta(G)$. If $\operatorname{smp}(G)=\delta(G)$, then $G$ is strongly maximally matched. In addition, for any strong matching preclusion set $F$, if $G-F$ has isolated vertices, then $G$ is strongly super matched and we say $F$ to be a trivial strong matching preclusion set, otherwise $F$ is nontrivial.

\subsection{Fractional(strong) Matching Preclusion}

A standard way to consider matchings in polyhedral combinatorics is as follows. Given a set of edges $S$ of $G$, we define $f^{S}$ to be the indicator function of $S$, that is, $f^{S}: E(G) \longrightarrow\{0,1\}$ such that $f_{S}(e)=1$ if and only if $e \in S$. Let $X$ be a set of vertices of $G$. We denote $\tau(X)$ to be the set of edges with exactly one end in $X$. If $X=\{v\}$, we write $\tau(v)$ instead of $\tau(\{v\})$. Clearly, $f^{M}: E(G) \longrightarrow\{0,1\}$ is the indicator function of the perfect matching $M$ if $\sum_{e \in \tau(v)} f^{M}(e)=1$ for each vertex $v$ of $G$, and $f^{M}: E(G) \longrightarrow\{0,1\}$ is the indicator function of the almost perfect matching $M$ if $\sum_{e \in \tau(v)} f^{M}(e)=1$ for each vertex $v$ of $G$, except one vertex say $v$, and $\sum_{e \in \tau(v)} f^{M}(e)=0$. In fact, $f^{M}(E(G))=|V(G)| / 2$ if $M$ is a perfect matching and $f^{M}(E(G))=(\mid V(G)-1) \mid / 2$ if $M$ is an almost perfect matching. A relaxation from an integer set to a continuous set is to replace the codomain of the indicator function from $\{0,1\}$ to the interval $[0,1]$. Let $f: E(G) \longrightarrow[0,1]$. Naturally, we call $f$ a fractional matching if $\sum_{e \in \tau(v)} f(e) \leq 1$ for each vertex $v$ of $G$. Similarly, $f$ is a fractional perfect matching if $\sum_{e \in \tau(v)} f(e)=1$ for each vertex $v$ of $G$. Thus, if $f$ is a fractional perfect matching, then

$$
f(E(G))=\sum_{e \in E(G)} f(e)=\frac{1}{2} \sum_{v \in V(G)} \sum_{e \sim v} f(e)=\frac{|V(G)|}{2} .
$$

Recently, Y. Liu and W. Liu (Y. Liu \& W. Liu, 2016) introduced such a generalization by precluding fractional perfect matchings only. An edge subset $F$ of $G$ is a fractional matching preclusion set (FMP set for short) if $G-F$ has no fractional perfect matchings. In addition, the fractional matching preclusion number (FMP number for short) of $G$, denoted by $f \operatorname{mp}(G)$, is the minimum size of $F M P$ sets of $G$, that is, $f \operatorname{mp}(G)=\min \{|F|:$ F is an FMP set $\}$. Clearly,

$$
f m p(G) \leq \delta(G)
$$

and by the definition of $\operatorname{fmp}(G)$, when $|V(G)|$ is even,

$$
m p(G) \leq f m p(G)
$$

If $\operatorname{fmp}(G)=\delta(G)$, then $G$ is fractional maximally matched. If $G-F$ has isolated vertices for every optimal fractional matching preclusion set $F$, then $G$ is fractional super matched.

Liu and Liu (Y. Liu \& W. Liu, 2016) also introduced a generalization of strong matching preclusion under the same assumption by precluding fractional perfect matchings only. A set $F$ of edges and vertices of $G$ is a fractional strong matching preclusion set (FSMP set for short) if $G-F$ has no fractional perfect matchings. The fractional strong matching preclusion number (FSMP number for short) of $G$, denoted by $f \operatorname{smp}(G)$, is the minimum size of FSMP sets of $G$, that is, $f \operatorname{smp}(G)=\min \{|F|: \mathrm{F}$ is an FSMP set $\}$. Since a fractional matching preclusion set is a fractional strong matching preclusion set, it is clear that

$$
f \operatorname{smp}(G) \leq f m p(G) \leq \delta(G)
$$




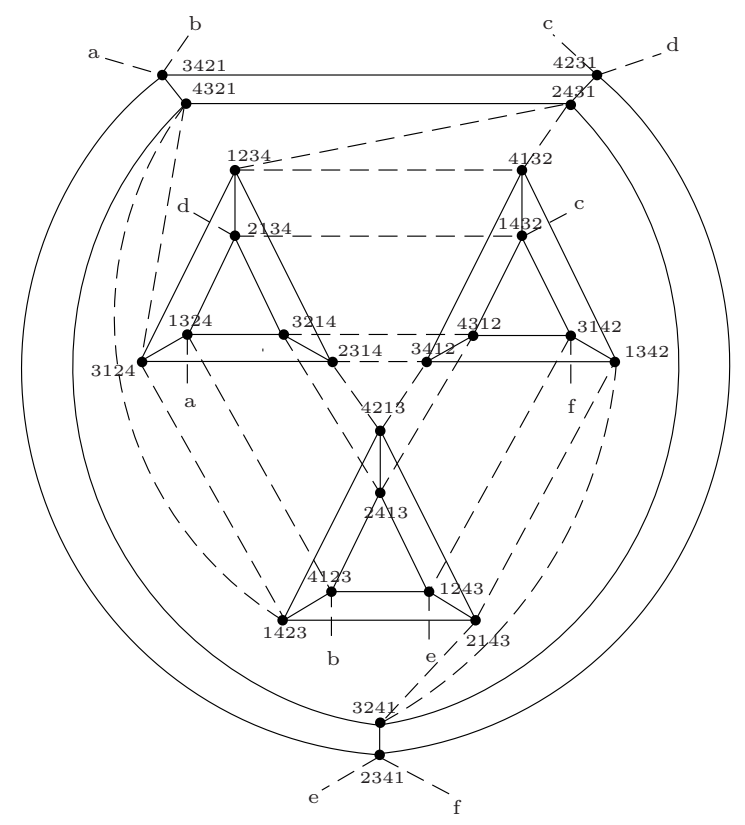

Figure 2. $S_{4}^{2}$

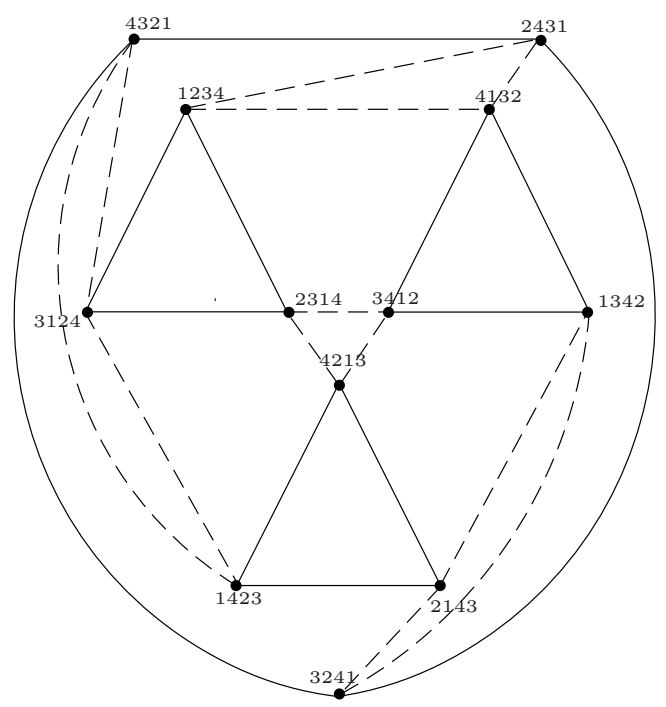

Figure 3. $A_{4}$

If $f \operatorname{smp}(G)=\delta(G)$, then $G$ is fractional strongly maximally matched. In addition, for any fractional strong matching preclusion set $F$, if $G-F$ has isolated vertices, then $G$ is fractional strongly super matched and we say $F$ to be a trivial fractional strong matching preclusion set, otherwise $F$ is nontrivial. For more details about this concept, we refer to the papers (Y. Liu \& W. Liu, 2016; Ma, Mao, Cheng, \& Wang, 2018).

\subsection{Split-Star Networks $S_{n}^{2}$}

The split-star network as a variant of the star-graph network. In particular, a split-star network can be decomposed into two disjoint alternating group networks. Both the star-graph and alternating group networks are special members of the family of generalized arrangement graphs (Day \& Tripathi, 1992; Jwo, Lakshmivarahan, \& Dhall, 1993). Therefore, the split-star network inherits the topological properties of the alternating group networks and arrangement graphs, and can be a good candidate for a multiprocessor interconnection.

In this paper, we study fractional strong matching preclusion problem for the split-star networks. We first give the definition of the split-star $S_{n}^{2}$ introduced in (Cheng, Lesniak, \& Park, 2001). The vertex set is the set of the $n$ ! permutations on $\{1,2, \cdots, n\}$. To describe the adjacency, it is convenient to look at the generator graph. Consider the generator graph 
in Figure 1 (a star with the root split). Two permutations are adjacent if one can be obtained from the other by either a 2-exchange or a 3-rotation. A 2-exchange interchanges the symbols in positions 1 and 2 (that is, corresponding to the center edge $(1,2)$ in Figure 1). A 3-rotation rotates the symbols in the positions labelled by the vertices of a triangle, that is, the triangle with vertices 1,2 and $k$ for some $k \in\{3,4, \cdots, n\}$. The rotation can be forward or reverse. So there are two 3-rotations for each $k$. Thus, $S_{n}^{2}$ is a $(2 n-3)$-regular graph with $n$ ! vertices. Throughout this paper, we use $\left[a_{1}, a_{2}, a_{3}, \cdots, a_{n}\right]$ to denote a permutation written as a rearrangement of objects, that is, $a_{i}$ in position $i$. However, for notational simplicity in pictures, this permutation is written as $a_{1} a_{2} a_{3} \cdots a_{n}$.

Let $V_{n}^{n: i}$ be the set of all vertices in $S_{n}^{2}$ with the $n$th position having value $i$, i.e., $V_{n}^{n: i}=\{p \mid p=\underbrace{x x \cdots x}_{n-1} i, x$ is a don't care symbol\}. The set $\left\{V_{n}^{n: i} \mid 1 \leq i \leq n\right\}$ forms a partition of $V\left(S_{n}^{2}\right)$. Let $S_{n-1}^{2: i}$ denote the subgraph of $S_{n}^{2}$ induced by $V_{n}^{n: i}$, i.e., $S_{n-1}^{2: i}=S_{n}^{2}\left[V_{n}^{n: i}\right]$, it is easy to know that $S_{n-1}^{2: i}$ is isomorphic to $S_{n-1}^{2}$. Every vertex $v$ in $S_{n-1}^{2: i}$ has exactly two neighbors outside of $S_{n-1}^{2: i}$; moreover these two neighbors belong to different $S_{n-1}^{2: j}$ s, where $j \neq i$. We call these neighbors as the external-neighbors of $v$. We call these edges, whose end-vertices belong to different subgraphs, as cross edges. For any two vertices in the same subgraph $S_{n-1}^{2: i}$, their external-neighbors in other subgraphs are different. For example, a partition of $S_{4}^{2}$ is shown in Figure 2. Let $S_{n, E}^{2}$ be a subgraph of $S_{n}^{2}$ induced by the set of even permutations. In other words, all even permutations form the vertex-set of $S_{n, E}^{2}$, in which the adjacency rule is precisely the 3-rotation. We know that $S_{n, E}^{2}$ is the alternating group graph $A_{n}$ (Jwo, Lakshmivarahan, Dhall, 1993). Let $S_{n, O}^{2}$ be a subgraph of $S_{n}^{2}$ induced by the set of all odd permutations, in which the adjacency rule is precisely the 3-rotation. We have that $S_{n, O}^{2}$ is isomorphic to $A_{n}$ and $S_{n, O}^{2}$ is isomorphic to $S_{n, E}^{2}$ via the 2-exchange $\phi\left(a_{1} a_{2} a_{3} \cdots a_{n}\right)=a_{2} a_{1} a_{3} \cdots a_{n}$. Hence, there are $\frac{n !}{2}$ matching edges between $S_{n, E}^{2}$ and $S_{n, O}^{2}$, i.e., there is one to one correspondence between $S_{n, E}^{2}$ and $S_{n, O}^{2}$. Indeed, the split-star network $S_{n}^{2}$ is introduced in (Cheng et al., 2001) which is the companion graph of $A_{n}$. In this paper, we study the fractional strong matching preclusion problem for the split-star graph. Because deletion of vertices is allowed, the analysis will be more involved than the analysis of the correspond matching preclusion problem.

\subsection{Related Results}

We summarize some knowledge which will be needed later.

Proposition 1 (Scheinerman \& Ullman, 1997) The graph $G$ has a fractional perfect matching if and only if there is a partition $\left\{V_{1}, V_{2}, \cdots, V_{n}\right\}$ of the vertex set of $V(G)$ such that, for each $i$, the graph $G\left[V_{i}\right]$ is either $K_{2}$ or a Hamiltonian graph on odd number of vertices.

lemma 1 (Ma, Mao, Cheng, \& Melekian, 2018.) Let $G$ be fractional strongly super matched graph with $\delta(G) \geq 2$. If $F$ is a trivial FSMP set of $G$ and $G-F$ has an isolated vertex $v$, then $G-F-v$ has a fractional perfect matching.

Before we prove the fractional strong perfect matching preclusion results of $S_{n}^{2}$, now we need some preliminary results on the perfect matching preclusion, strong perfect matching preclusion and Hamiltonian properties of graphs $S_{n}^{2}$ and $A_{n}$.

Theorem 1 (Hsu, Li, Tan, \& Hsu, 2004) Let $n \geq 4$. Suppose $F \subseteq V\left(A_{n}\right) \cup E\left(A_{n}\right)$. If $|F| \leq 2 n-7$, then $A_{n}-F$ is Hamiltonian connected; if $|F| \leq 2 n-6$, then $A_{n}-F$ is Hamiltonian.

Theorem 2 (Cheng, Lesniak, Lipman, \& Lipták, 2008) Suppose $n \geq 4$. Then $m p\left(A_{n}\right)=2 n-4$ and $m p\left(S_{n}^{2}\right)=2 n-3$. Moreover, every optimal matching preclusion set is trivial.

Theorem 3 (Bonneville, Cheng, \& Renzi, 2011) Let $n \geq 4$. Then $\operatorname{smp}\left(A_{n}\right)=2 n-4$. Moreover $A_{n}$ is super strongly matched; that is, every optimal strong matching preclusion set of $A_{n}$ is trivial.

Corollary 1 (Bonneville, Cheng, \& Renzi, 2011) Let $S_{n}^{2}$ be a split-star with $n \geq 4$. Then $S_{n}^{2}$ is maximally strongly matched; that is, $\operatorname{smp}\left(S_{n}^{2}\right)=2 n-3$. Moreover, $S_{n}^{2}$ is super strongly matched; that is, every optimal strong matching preclusion set of $S_{n}^{2}$ is trivial.

lemma 2 (Cheng \& Siddiqui, 2016) Suppose $G$ has an almost perfect matching $M$ missing $v$. If $v$ is not an isolated vertex in $G$, then $G$ has an almost perfect matching missing a vertex other than $v$.

\section{Main Results}

Since $S_{n}^{2}$ has an even number of vertices and is $(2 n-3)$-regular, the next result follows by Theorem 2 and $m p\left(S_{n}^{2}\right) \leq$ $\operatorname{fmp}\left(S_{n}^{2}\right) \leq \delta\left(S_{n}^{2}\right)$.

Theorem 4 Let $n \geq 4$. Then $\operatorname{fmp}\left(S_{n}^{2}\right)=2 n-3$. Moreover, $S_{n}^{2}$ is fractional super matched.

We now turn our attention to $S_{n}^{2}$. Note that $S_{n-1}^{2: i}$ is isomorphic to $S_{n-1}^{2}$, where $1 \leq i \leq n$. it is easy to know that $S_{n}^{2}$ can be decomposed into $n$ copies, i.e., $S_{n-1}^{2: 1}, S_{n-1}^{2: 2}, \cdots, S_{n-1}^{2: n}$. We will prove a more general result. 
Theorem 5 If $S_{n-1}^{2}$ is fractional strongly super matched for $n \geq 5$, then $S_{n}^{2}$ is fractional strongly super matched.

Proof. Let $S_{n}^{2}$ be a graph that consists of $G_{1}, G_{2}, \cdots, G_{n}$, where $G_{1}, G_{2}, \cdots, G_{n}$ are copies of $S_{n-1}^{2}$. Let $F \subseteq E\left(S_{n}^{2}\right) \cup V\left(S_{n}^{2}\right)$ with $|F| \leq 2 n-3$. Let $F_{i}=G_{i} \cap F$, where $1 \leq i \leq n$. For notational convenience, we assume $\left|F_{i}\right| \leq\left|F_{1}\right|$ for $2 \leq i \leq n$. We will show that $S_{n}^{2}-F$ satisfy one of the following: (1) $S_{n}^{2}-F$ has a fractional perfect matching; (2) $S_{n}^{2}-F$ has an isolated vertex such that $F$ is trivial FSMP set. If (2) is true, then we are done. So we may assume that $S_{n}^{2}-F$ has no isolated vertices.

Case 1. $\left|F_{1}\right|=2 n-3$. Clearly, $\left|F_{i}\right|=0$ for each $F_{i}$, where $2 \leq i \leq n$. Let $F_{1}^{\prime}=F_{1}-\{\alpha, \beta\}$, where $\{\alpha, \beta\} \subseteq F_{1}$. Since $G_{1}$ is fractional strongly super matched, $G_{1}-F_{1}^{\prime}$ has a fractional perfect matching or $F_{1}^{\prime}$ is a trivial FSMP set of $G_{1}$. If $G_{1}-F_{1}^{\prime}$ has a fractional perfect matching, we consider to delete elements $\alpha$ and $\beta$ from $G_{1}-F_{1}^{\prime}$ and construct a fractional perfect matching of $S_{n}^{2}-F$. If $F_{1}^{\prime}$ is a trivial FSMP set of $G_{1}$ and $v$ is an isolated vertex of $G_{1}-F_{1}^{\prime}$, then $G_{1}^{\prime}=G_{1}-F_{1}^{\prime}-\{v\}$ has fractional perfect matching by Lemma 1. Similarly, we consider to delete elements $\alpha, \beta$ and $v$ from $G_{1}-F_{1}^{\prime}$ and construct a fractional perfect matching of $S_{n}^{2}-F$. Compared with the case that $F_{1}^{\prime}$ is a trivial FSMP set of $G_{1}$, the case that $G_{1}-F_{1}^{\prime}$ has a fractional perfect matching is easy and clear to construct a fractional perfect matching of $S_{n}^{2}-F$. Therefore we consider the difficult case that $F_{1}^{\prime}$ is a trivial FSMP set of $G_{1}$. We can easily see that it is possible that $v \in\{\alpha, \beta\}$. If $v \in\{\alpha, \beta\}$, we only consider to delete elements $\alpha$ and $\beta$ from $G_{1}-F_{1}^{\prime}$ and this is easier to construct a fractional perfect matching than deleting elements $\alpha, \beta$ and $v$ from $G_{1}-F_{1}^{\prime}$. So we only consider the case deleting elements $\alpha, \beta$ and $v$ from $G_{1}-F_{1}^{\prime}$ in the following. Since $G_{1}^{\prime}$ has a fractional perfect matching, it follows from Proposition 1 that there is a partition $\left\{V_{1}, V_{2}, \cdots, V_{t}\right\}$ of the vertex set of $V\left(G_{1}^{\prime}\right)$ such that, for each $i$, the graph $G_{1}^{\prime}\left[V_{i}\right]$ is either graph $K_{2}$ or a Hamiltonian graph on odd number of vertices. To show that $S_{n}^{2}-F$ has a fractional perfect matching, we consider the following cases according to elements $\alpha$ and $\beta$.

Subcase 1.1. $\alpha$ and $\beta$ are two vertices. If $\alpha$ and $\beta$ are two vertices of $K_{2}$ induced by $V_{i}$, note that there exists a partition $\left\{V_{1}^{\prime}, V_{2}^{\prime}, \cdots, V_{t-1}^{\prime}\right\}$ of the vertex set of $V\left(G_{1}^{\prime}-\{\alpha, \beta\}\right)$ such that, $G_{1}^{\prime}-\{\alpha, \beta\}$ has a fractional perfect matching $f_{1}$ by Proposition 1. Let $F_{i}^{\prime}=\left\{v^{\prime}\right\} \cap V\left(G_{i}\right)$ for $2 \leq i \leq n$, where $v^{\prime}$ is external-neighbor of $v$. Since $\left|F_{i}^{\prime}\right| \leq 1$ and $G_{i}$ is $(2 n-5)$-regular for $2 \leq i \leq n, G_{i}-F_{i}^{\prime}$ has a fractional perfect matching $f_{i}$. Thus $\left\{\left(v v^{\prime}\right)\right\}$ and $f_{1}, f_{2}, \cdots, f_{n}$ induce a fractional perfect matching of $S_{n}^{2}-F$. If $\alpha$ is a vertex of $K_{2}$ induced by $V_{i}$ and $\beta$ is a vertex of $K_{2}$ induced by $V_{j}$, where $i \neq j$, there exists a fractional perfect matching $f_{1}$ in $G_{1}^{\prime}-\{\alpha, \beta, \gamma, \lambda\}$, where $\gamma$ is a neighbor of $\alpha$ in $K_{2}$ induced by $V_{i}$ and $\lambda$ is a neighbor of $\beta$ in $K_{2}$ induced by $V_{j}$. Since every vertex in $G_{1}$ has exactly two external-neighbors in $S_{n}^{2}-G_{1}$ and these two neighbors belong to different $G_{i}$, where $2 \leq i \leq n$, it follows that we may select an external-neighbor for each vertex from $\{\gamma, \lambda\}$ such that they are in different $G_{i}$ 's. Let $F_{i}^{\prime}=\left\{\gamma^{\prime}, \lambda^{\prime}, v^{\prime}\right\} \cap V\left(G_{i}\right)$ for $2 \leq i \leq n$, where $\gamma^{\prime}, \lambda^{\prime}$ and $v^{\prime}$ are external-neighbors of $\gamma, \lambda$ and $v$, respectively. Note that $\left|F_{i}^{\prime}\right| \leq 2$ and $G_{i}$ is $(2 n-5)$-regular, so $G_{i}-F_{i}^{\prime}$ has a fractional perfect matching $f_{i}$, where $2 \leq i \leq n$. Thus, $\left\{\left(\gamma \gamma^{\prime}\right),\left(\lambda \lambda^{\prime}\right),\left(v v^{\prime}\right)\right\}$ and $f_{1}, f_{2}, \cdots, f_{n}$ induce a fractional perfect matching of $S_{n}^{2}-F$. If $\alpha$ and $\beta$ are two vertices of a Hamiltonian graph on odd number of vertices induced by $V_{i}$, then there is a fractional perfect matching $f_{1}$ in $G_{1}^{\prime}-\{\alpha, \beta, \gamma\}$ by Proposition 1, where $\gamma$ is a neighbor of $\alpha$ in an odd path of $G_{1}^{\prime}-\{\alpha, \beta\}$. Let $F_{i}^{\prime}=\left\{\gamma^{\prime}, v^{\prime}\right\} \cap V\left(G_{i}\right)$ for $2 \leq i \leq n$, where $\gamma^{\prime}$ and $v^{\prime}$ are external-neighbors of $\gamma$ and $v$, respectively. Note that $\left|F_{i}^{\prime}\right| \leq 2$ and $G_{i}$ is $(2 n-5)$-regular, so $G_{i}-F_{i}^{\prime}$ has a fractional perfect matching $f_{i}$, where $2 \leq i \leq n$. Hence, $\left\{\left(v v^{\prime}\right),\left(\gamma \gamma^{\prime}\right)\right\}$ and $f_{1}, f_{2}, \cdots, f_{n}$ induce a fractional perfect matching of $S_{n}^{2}-F$. If $\alpha$ and $\beta$ are two vertices of two Hamiltonian graphs on odd number of vertices induced by $V_{i}$ and $V_{j}$, respectively, where $i \neq j$, then there is a fractional perfect matching $f_{1}$ in $G_{1}-F_{1}-\{v\}$ by Lemma 1 . Let $F_{i}^{\prime}=\left\{v^{\prime}\right\} \cap V\left(G_{i}\right)$ for $2 \leq i \leq n$, where $v^{\prime}$ is external-neighbor of $v$. Since $\left|F_{i}^{\prime}\right| \leq 1$ and $G_{i}$ is $(2 n-3)$-regular for $2 \leq i \leq n$, $G_{i}-F_{i}^{\prime}$ has a fractional perfect matching $f_{i}$. Thus, $\left\{\left(v v^{\prime}\right)\right\}$ and $f_{1}, f_{2}, \cdots, f_{n}$ induce a fractional perfect matching of $S_{n}^{2}-F$. If $\alpha$ is a vertex of $K_{2}$ induced by $V_{i}$ and $\beta$ is a vertex of Hamiltonian graph on odd number of vertices induced by $V_{j}$, where $i \neq j$. There exists a fractional perfect matching $f_{1}$ in $G_{1}^{\prime}-\{\alpha, \beta, \gamma\}$, where $\gamma$ is neighbor of $\alpha$ in $K_{2}$ induced by $V_{i}$. Note that there are two vertices $\gamma$ and $v$ in $G_{1}-F_{1}$ that are unmatched, so let $F_{i}^{\prime}=\left\{\gamma^{\prime}, v^{\prime}\right\} \cap V\left(G_{i}\right)$ for $2 \leq i \leq n$, where $\gamma^{\prime}$ and $v^{\prime}$ are external-neighbors of $\gamma$ and $v$, respectively. Note that $\left|F_{i}^{\prime}\right| \leq 2$ and $G_{i}$ is $(2 n-5)$-regular, so $G_{i}-F_{i}^{\prime}$ has a fractional perfect matching $f_{i}$, where $2 \leq i \leq n$. Thus, $\left\{\left(v v^{\prime}\right),\left(\gamma \gamma^{\prime}\right)\right\}$ and $f_{1}, f_{2}, \cdots, f_{n}$ induce a fractional perfect matching of $S_{n}^{2}-F$.

Subcase 1.2. $\alpha$ is a vertex and $\beta$ is an edge. Let $\beta=u w$. Suppose $\alpha$ is a vertex of $K_{2}$ induced by $V_{i}$ and $\beta$ is an edge of $K_{2}$ induced by $V_{i}$. Without loss of generality, we assume $\alpha=u$. For $i=1$, there is a fractional perfect matching $f_{1}$ in $G_{1}^{\prime}-\{\alpha, \beta, w\}$ by Proposition 1. Note that there are two vertices $w$ and $v$ in $G_{1}-F_{1}$ that are unmatched, so let $F_{i}^{\prime}=\left\{w^{\prime}, v^{\prime}\right\} \cap V\left(G_{i}\right)$ for $2 \leq i \leq n$, where $w^{\prime}$ and $v^{\prime}$ are external-neighbors of $w$ and $v$, respectively. For $2 \leq i \leq n$, $\left|F_{i}^{\prime}\right| \leq 2$ and $G_{i}$ is $(2 n-5)$-regular, so $G_{i}-F_{i}^{\prime}$ has a fractional perfect matching $f_{i}$. Hence, $\left\{\left(w w^{\prime}\right),\left(v v^{\prime}\right)\right\}$ and $f_{1}, f_{2}, \cdots, f_{n}$ induce a fractional perfect matching of $S_{n}^{2}-F$. Suppose $\alpha$ is a vertex of $K_{2}$ induced by $V_{i}$, and $\beta$ is an edge of $K_{2}$ induced by $V_{j}$, where $i \neq j$. Note that there exists a partition $\left\{V_{1}^{\prime}, V_{2}^{\prime}, \cdots, V_{t-2}^{\prime}\right\}$ of the vertex set of $V\left(G_{1}^{\prime}-\{\alpha, \beta, \gamma, u, w\}\right)$ such that, there is a fractional perfect matching $f_{1}$ in $G_{1}^{\prime}-\{\alpha, \beta, \gamma, u, w\}$ by Proposition 1, where $\gamma$ is a neighbor of $\alpha$ in $K_{2}$ induced by $V_{i}$. Since every vertex in $G_{1}$ has exactly two neighbors outside in $S_{n}^{2}-G_{1}$; moreover, these two neighbors belong to different $G_{i}$, where $2 \leq i \leq n$, it follows that we may select an external-neighbor for each vertex from $\{\gamma, u, w\}$ 
such that they are in different $G_{i}$ 's. Note that there are four vertices $\gamma, u, w$ and $v$ in $G_{1}-F_{1}$ that are unmatched, so let $F_{i}^{\prime}=\left\{\gamma^{\prime}, u^{\prime}, w^{\prime}, v^{\prime}\right\} \cap V\left(G_{i}\right)$ for $2 \leq i \leq n$, where $\gamma^{\prime}, u^{\prime}, w^{\prime}$ and $v^{\prime}$ are external-neighbors of $\gamma, u, w$ and $v$, respectively. For $2 \leq i \leq n,\left|F_{i}^{\prime}\right| \leq 2$ and $G_{i}$ is $(2 n-5)$-regular, so $G_{i}-F_{i}^{\prime}$ has a fractional perfect matching $f_{i}$ for $2 \leq i \leq n$. Hence, $\left\{\left(\gamma \gamma^{\prime}\right),\left(u u^{\prime}\right),\left(w w^{\prime}\right),\left(v v^{\prime}\right)\right\}$ and $f_{1}, f_{2}, \cdots, f_{n}$ induce a fractional perfect matching of $S_{n}^{2}-F$. Suppose vertex $\alpha$ and edge $\beta$ are of a Hamiltonian graph on odd number of vertices induced by $V_{i}$. There exists a fractional perfect matching $f_{1}$ missing at most two vertices $x$ and $y$ in $G_{1}^{\prime}-\{x, y, \alpha, \beta\}$, where $x$ and $y$ are two vertices of a Hamiltonian graph on odd number of vertices induced by $V_{i}$. We may select an external-neighbor for each vertex from $\{x, y\}$ such that they are in different $G_{i}$ 's, where $2 \leq i \leq n$. Let $F_{i}^{\prime}=\left\{x^{\prime}, y^{\prime}, v^{\prime}\right\} \cap V\left(G_{i}\right)$ for $2 \leq i \leq n$, where $x^{\prime}, y^{\prime}$ and $v^{\prime}$ are external-neighbors of $x, y$ and $v$, respectively. For $2 \leq i \leq n,\left|F_{i}^{\prime}\right| \leq 2$ and $G_{i}$ is $(2 n-5)$-regular, so $G_{i}-F_{i}^{\prime}$ has a fractional perfect matching $f_{i}$. Hence, $\left\{\left(x x^{\prime}\right),\left(y y^{\prime}\right),\left(v v^{\prime}\right)\right\}$ and $f_{1}, f_{2}, \cdots, f_{n}$ induce a fractional perfect matching of $S_{n}^{2}-F$. Suppose $\alpha$ is a vertex of $K_{2}$ induced by $V_{i}$ and $\beta$ is an edge of a Hamiltonian graph on odd number of vertices induced by $V_{j}$, where $i \neq j$. Note that $G_{1}^{\prime}-\{\alpha, \beta, \gamma, u\}$ has a fractional perfect matching $f_{1}$, where $\gamma$ is a neighbor of $\alpha$ in $K_{2}$ induced by $V_{i}$ and vertex $u$ is incident with $\beta$ in the Hamiltonian graph on odd number of vertices induced by $V_{j}$. Since every vertex in $G_{1}$ has exactly two neighbors outside in $S_{n}^{2}-G_{1}$; moreover, these two neighbors belong to different $G_{i}$, where $2 \leq i \leq n$, it follows that we may select an external-neighbor for each vertex from $\{\gamma, u\}$ such that they are in different $G_{i}$ 's. Note that there are three vertices $\gamma, u$ and $v$ in $G_{1}-F_{1}$ that are unmatched, so let $F_{i}^{\prime}=\left\{\gamma^{\prime}, u^{\prime}, v^{\prime}\right\} \cap V\left(G_{i}\right)$ for $2 \leq i \leq n$, where $\gamma^{\prime}, u^{\prime}$ and $v^{\prime}$ are external-neighbors of $\gamma, u$ and $v$, respectively. For $2 \leq i \leq n,\left|F_{i}^{\prime}\right| \leq 2$ and $G_{i}$ is $(2 n-5)$-regular, so $G_{i}-F_{i}^{\prime}$ has a fractional perfect matching $f_{i}$. Thus, $\left\{\left(\gamma \gamma^{\prime}\right),\left(u u^{\prime}\right),\left(v v^{\prime}\right)\right\}$ and $f_{1}, f_{2}, \cdots, f_{n}$ induce a fractional perfect matching of $S_{n}^{2}-F$. Suppose $\alpha$ is a vertex of a Hamiltonian graph on odd number of vertices induced by $V_{i}$ and $\beta$ is an edge of $K_{2}$ induced by $V_{j}$, where $i \neq j$. Note that $G_{1}^{\prime}-\{\alpha, \beta, u, w\}$ has a fractional perfect matching $f_{1}$, where $u$ and $w$ are incident with $\beta$. We may select an external-neighbor for each vertex from $\{u, w\}$ such that they are in different $G_{i}$ 's, where $2 \leq i \leq n$. Let $F_{i}^{\prime}=\left\{u^{\prime}, w^{\prime}, v^{\prime}\right\} \cap V\left(G_{i}\right)$ for $2 \leq i \leq n$, where $u^{\prime}, w^{\prime}$ and $v^{\prime}$ are external-neighbors of $u, w$ and $v$, respectively. For $2 \leq i \leq n,\left|F_{i}^{\prime}\right| \leq 2$ and $G_{i}$ is $(2 n-5)$-regular, so $G_{i}-F_{i}^{\prime}$ has a fractional perfect matching $f_{i}$. Thus, $\left\{\left(u u^{\prime}\right),\left(w w^{\prime}\right),\left(v v^{\prime}\right)\right\}$ and $f_{1}, f_{2}, \cdots, f_{n}$ induce a fractional perfect matching of $S_{n}^{2}-F$.

Subcase 1.3. $\alpha$ and $\beta$ are two edges. Let $\alpha=x y$ and $\beta=u w$. Suppose $\alpha$ is an edge of $K_{2}$ induced by $V_{i}$ and $\beta$ is an edge of $K_{2}$ induced by $V_{j}$, where $i \neq j$. There is a fractional perfect matching $f_{1}$ in $G_{1}^{\prime}-\{\alpha, \beta, x, y, u, w\}$ by Proposition 1 , where $x$ and $y$ are incident with $\alpha$, and $u$ and $w$ are incident with $\beta$. Every vertex in $G_{1}$ has exactly two neighbors outside in $S_{n}^{2}-G_{1}$; moreover, these two neighbors belong to different $G_{i}$, where $2 \leq i \leq n$. So we may select an external-neighbor for each vertex from $\{x, y, u, w\}$ such that they are in different $G_{i}$ 's. Let $F_{i}^{\prime}=\left\{x^{\prime}, y^{\prime}, u^{\prime}, w^{\prime}, v^{\prime}\right\} \cap V\left(G_{i}\right)$ for $2 \leq i \leq n$, where $x^{\prime}, y^{\prime}, u^{\prime}, w^{\prime}$ and $v^{\prime}$ are external-neighbors of $x, y, u, w$ and $v$, respectively. For $2 \leq i \leq n$, since $\left|F_{i}^{\prime}\right| \leq 2$ and $G_{i}$ is $(2 n-5)$-regular, it follows that $G_{i}-F_{i}^{\prime}$ has a fractional perfect matching $f_{i}$. Therefore, $\left\{\left(x x^{\prime}\right),\left(y y^{\prime}\right),\left(u u^{\prime}\right),\left(w w^{\prime}\right),\left(v v^{\prime}\right)\right\}$ and $f_{1}, f_{2}, \cdots, f_{n}$ induce a fractional perfect matching of $S_{n}^{2}-F$. Suppose edge $\alpha$ and edge $\beta$ are of a Hamiltonian graph on odd number of vertices induced by $V_{i}$. There is a fractional perfect matching $f_{1}$ in $G_{1}^{\prime}-\{\alpha, \beta\}$ missing vertex $k$. Note that there are two vertices $k$ and $v$ in $G_{1}-F_{1}$ that are unmatched, so let $F_{i}^{\prime}=\left\{k^{\prime}, v^{\prime}\right\} \cap V\left(G_{i}\right)$ for $2 \leq i \leq n$, where $k^{\prime}$ and $v^{\prime}$ are external-neighbors of $k$ and $v$, respectively. For $2 \leq i \leq n,\left|F_{i}^{\prime}\right| \leq 2$ and $G_{i}$ is $(2 n-5)$-regular. So $G_{i}-F_{i}^{\prime}$ has a fractional perfect matching $f_{i}$ for $2 \leq i \leq n$. Thus, $\left\{\left(k k^{\prime}\right),\left(v v^{\prime}\right)\right\}$ and $f_{1}, f_{2}, \cdots, f_{n}$ induce a fractional perfect matching of $S_{n}^{2}-F$. Suppose $\alpha$ is an edge of $K_{2}$ induced by $V_{i}$ and $\beta$ is an edge Hamiltonian graph on odd number of vertices induced by $V_{j}$, where $i \neq j$. There exists a fractional perfect matching $f_{1}$ in $G_{1}^{\prime}-\{\alpha, \beta, x, y, u\}$, where $x$ and $y$ are incident with $\alpha$ and $u$ is incident with $\beta$. Every vertex in $G_{1}$ has exactly two neighbors outside in $S_{n}^{2}-G_{1}$; moreover, these two neighbors belong to different $G_{i}$, where $2 \leq i \leq n$. So we may select an external-neighbor for each vertex from $\{x, y, u\}$ such that they are in different $G_{i}{ }^{\prime}$ s. Let $F_{i}^{\prime}=\left\{x^{\prime}, y^{\prime}, u^{\prime}, v^{\prime}\right\} \cap V\left(G_{i}\right)$ for $2 \leq i \leq n$, where $x^{\prime}, y^{\prime}, u^{\prime}$ and $v^{\prime}$ are external-neighbors of $x$, $y, u$ and $v$, respectively. For $2 \leq i \leq n,\left|F_{i}^{\prime}\right| \leq 2$ and $G_{i}$ is $(2 n-5)$-regular. So $G_{i}-F_{i}^{\prime}$ has a fractional perfect matching $f_{i}$ for $2 \leq i \leq n$. Therefore, $\left\{\left(x x^{\prime}\right),\left(y y^{\prime}\right),\left(u u^{\prime}\right),\left(v v^{\prime}\right)\right\}$ and $f_{1}, f_{2}, \cdots, f_{n}$ induce a fractional perfect matching of $S_{n}^{2}-F$.

Case 2. $\left|F_{1}\right|=2 n-4$. Clearly, $\left|F_{i}\right| \leq 1$ for $2 \leq i \leq n$. Let $F_{1}^{\prime}=F_{1}-\{\alpha\}$, where $\alpha \in F_{1}$. Since $G_{1}$ is fractional strongly super matched, it follows that either graph $G_{1}-F_{1}^{\prime}$ has a fractional perfect matching or $F_{1}^{\prime}$ is a trivial FSMP set. If $G_{1}-F_{1}^{\prime}$ has a fractional perfect matching, we consider to delete element $\alpha$ from $G_{1}-F_{1}^{\prime}$ and construct a fractional perfect matching of $S_{n}^{2}-F$. If $F_{1}^{\prime}$ is a trivial FSMP set of $G_{1}$ and $v$ is an isolated vertex of $G_{1}-F_{1}^{\prime}$, then $G_{1}^{\prime}=G_{1}-F_{1}^{\prime}-\{v\}$ has fractional perfect matching by Lemma 1 . Similarly, we consider to delete elements $\alpha$ and $v$ from $G_{1}-F_{1}^{\prime}$ and construct a fractional perfect matching of $S_{n}^{2}-F$. Compared with the case that $F_{1}^{\prime}$ is a trivial FSMP set of $G_{1}$, the case that $G_{1}-F_{1}^{\prime}$ has a fractional perfect matching is easy and clear to construct a fractional perfect matching of $S_{n}^{2}-F$. Therefore we consider the difficult case that $F_{1}^{\prime}$ is a trivial FSMP set of $G_{1}$. We can easily see that it is possible that $v=\alpha$. If $v=\alpha$, we only consider to delete element $\alpha$ from $G_{1}-F_{1}^{\prime}$ and this is easier to construct a fractional perfect matching than deleting elements $\alpha$ and $v$ from $G_{1}-F_{1}^{\prime}$. So we only consider the case deleting elements $\alpha$ and $v$ from $G_{1}-F_{1}^{\prime}$ in the following. Since $G_{1}^{\prime}$ has a fractional perfect matching, it follows from Proposition 1 that there is a partition $\left\{V_{1}, V_{2}, \cdots, V_{t}\right\}$ of the vertex set of $V\left(G_{1}^{\prime}\right)$ such that, for each $i$, the graph $G_{1}^{\prime}\left[V_{i}\right]$ is either graph $K_{2}$ or a Hamiltonian graph on odd number of vertices. To show that $S_{n}^{2}-F$ has a fractional perfect matching, we consider the following cases according to element $\alpha$. 
Subcase 2.1. $\alpha$ is a vertex. Suppose $\alpha$ is a vertex of $K_{2}$ induced by $V_{i}$. Note that there exists a partition $\left\{V_{1}^{\prime}, V_{2}^{\prime}, \cdots, V_{t-1}^{\prime}\right\}$ of the vertex set of $V\left(G_{1}^{\prime}-\{\alpha, \gamma\}\right)$ such that, for each $i$, the graph $\left(G_{1}^{\prime}-\{\alpha, \gamma\}\right)\left[V_{i}\right]$ is either graph $K_{2}$ or a Hamiltonian graph on odd number of vertices by Proposition 1, where $\gamma$ is a neighbor of $\alpha$ in $K_{2}$ induced by $V_{i}$. There is a fractional perfect matching $f_{1}$ in $G_{1}^{\prime}-\{\alpha, \gamma\}$ by Proposition 1 . Note that there are two vertices $\gamma$ and $v$ in $G_{1}-F_{1}$ that are unmatched, so let $F_{i}^{\prime}=\left(\left\{\gamma^{\prime}, v^{\prime}\right\} \cap V\left(G_{i}\right)\right) \cup F_{i}$, where $\gamma^{\prime}$ and $v^{\prime}$ are external-neighbors of $\gamma$ and $v$ in different $G_{i}$ 's, respectively. Note that $\left|F_{i}^{\prime}\right| \leq 2$ and $G_{i}$ is $(2 n-5)$-regular, so $G_{i}-F_{i}^{\prime}$ has a fractional perfect matching $f_{i}$, where $2 \leq i \leq n$. Hence, $\left\{\left(\gamma \gamma^{\prime}\right),\left(v v^{\prime}\right)\right\}$ and $f_{1}, f_{2}, \cdots, f_{n}$ induce a fractional perfect matching of $S_{n}^{2}-F$. Suppose $\alpha$ is a vertex of a Hamiltonian graph on odd number of vertices. It is obvious that $G_{1}-F_{1}-\{v\}$ has a fractional perfect matching $f_{1}$. Note that there is one vertex $v$ in $G_{1}-F_{1}$ that is unmatched, so let $F_{i}^{\prime}=\left(\left\{v^{\prime}\right\} \cap V\left(G_{i}\right)\right) \cup F_{i}$ for $2 \leq i \leq n$, where $v^{\prime}$ is external-neighbor of $v$. This facts imply that vertex $v$ can be matched to vertex $v^{\prime}$. Since $\left|F_{i}^{\prime}\right| \leq 2$ and $G_{i}$ is $(2 n-5)$-regular for $2 \leq i \leq n, G_{i}-F_{i}^{\prime}$ has a fractional perfect matching $f_{i}$. Thus, $\left\{\left(v v^{\prime}\right)\right\}$ and $f_{1}, f_{2}, \cdots, f_{n}$ induce a fractional perfect matching of $S_{n}^{2}-F$.

Subcase 2.2. $\alpha$ is an edge. Let $\alpha=u w$. Suppose $\alpha$ is an edge of $K_{2}$ induced by $V_{i}$. Note that there exists a partition $\left\{V_{1}^{\prime}, V_{2}^{\prime}, \cdots, V_{t-1}^{\prime}\right\}$ of the vertex set of $V\left(G_{1}^{\prime}-\{\alpha, u, w\}\right)$ such that, for each $i$, the graph $\left(G_{1}^{\prime}-\{\alpha, u, w\}\right)\left[V_{i}\right]$ is either graph $K_{2}$ or a Hamiltonian graph on odd number of vertices by Proposition 1. For $i=1$, there is a fractional perfect matching $f_{1}$ in $G_{1}^{\prime}-\{\alpha, u, w\}$ by Proposition 1 . For $2 \leq i \leq n,\left|F_{i}\right| \leq 1$. Every vertex in $G_{1}$ has exactly two neighbors outside in $S_{n}^{2}-G_{1}$; moreover, these two neighbors belong to different $G_{i}$, where $2 \leq i \leq n$. So we may select an external-neighbor for each vertex from $\{u, w, v\}$ such that they are in different $G_{i}$ 's. Let $F_{i}^{\prime}=\left(\left\{u^{\prime}, w^{\prime}, v^{\prime}\right\} \cap V\left(G_{i}\right)\right) \cup F_{i}$ for $2 \leq i \leq n$, where $u^{\prime}, w^{\prime}$ and $v^{\prime}$ are external-neighbors of $u, w$ and $v$ in different $G_{i}$ 's, respectively. Note that $\left|F_{i}^{\prime}\right| \leq 2$ and $G_{i}$ is $(2 n-5)$-regular, so $G_{i}-F_{i}^{\prime}$ has a fractional perfect matching $f_{i}$, where $2 \leq i \leq n$. Thus, $\left\{\left(u u^{\prime}\right),\left(w w^{\prime}\right),\left(v v^{\prime}\right)\right\}$ and $f_{1}, f_{2}, \cdots, f_{n}$ induce a fractional perfect matching of $S_{n}^{2}-F$. Suppose $\alpha$ is an edge of a Hamiltonian graph on odd number of vertices. It is obvious that there exists a Hamiltonian path $V_{i}-\{u\}$ on even number of vertices. Note that there exists a partition $\left\{V_{1}^{\prime}, V_{2}^{\prime}, \cdots, V_{m}^{\prime}\right\}$ of the vertex set of $V\left(G_{1}^{\prime}-\{\alpha, u\}\right)$ such that, for each $i$, the graph $\left(G_{1}^{\prime}-\{\alpha, u\}\right)\left[V_{i}\right]$ is either graph $K_{2}$ or a Hamiltonian graph on odd number of vertices by Proposition 1. For $i=1$, there is a fractional perfect matching $f_{1}$ in $G_{1}^{\prime}-\{\alpha, u\}$ by Proposition 1. Let $F_{i}^{\prime}=\left\{u^{\prime}, v^{\prime}\right\} \cap V\left(G_{i}\right)$, where $u^{\prime}$ and $v^{\prime}$ are external-neighbors of $u$ and $v$ in different $G_{i}$ 's, respectively. Note that $\left|F_{i}^{\prime}\right| \leq 2$ and $G_{i}$ is $(2 n-5)$-regular, so $G_{i}-F_{i}^{\prime}$ has a fractional perfect matching $f_{i}$, where $2 \leq i \leq n$. Hence, $\left\{\left(u u^{\prime}\right),\left(v v^{\prime}\right)\right\}$ and $f_{1}, f_{2}, \cdots, f_{n}$ induce a fractional perfect matching of $S_{n}^{2}-F$.

Case 3. $\left|F_{1}\right|=2 n-5$. Clearly, $\left|F_{i}\right| \leq 2$ for $2 \leq i \leq n$. $G_{1}$ is fractional strongly super matched, which implies that either graph $G_{1}-F_{1}$ has a fractional perfect matching or $F_{1}$ is a trivial FSMP set. Suppose $G_{1}-F_{1}$ has a fractional perfect matching $f_{1}$. For $2 \leq i \leq n$, note that $\left|F_{i}\right| \leq 2$ and $G_{i}$ is $(2 n-5)$-regular, so $G_{i}-F_{i}$ has a fractional perfect matching $f_{i}$. Thus $f_{1}, f_{2}, \cdots, f_{n}$ induce a fractional perfect matching of $S_{n}^{2}-F$. Suppose $F_{1}$ is a trivial FSMP set and $v$ is isolated vertex of $G_{1}-F_{1}$. For $2 \leq i \leq n,\left|F_{i}\right| \leq 2$. Let $F_{i}^{\prime}=\left\{v^{\prime}\right\} \cap V\left(G_{i}\right) \cup F_{i}$, where $v^{\prime}$ is an external-neighbor of $v$. Note that $\left|F_{i}^{\prime}\right| \leq 3$ and $G_{i}$ is $(2 n-5)$-regular, so $G_{i}-F_{i}^{\prime}$ has a fractional perfect matching $f_{i}$. Thus, $\left\{\left(v v^{\prime}\right)\right\}$ and $f_{1}, f_{2}, \cdots, f_{n}$ induce a fractional perfect matching of $S_{n}^{2}-F$.

Case 4. $\left|F_{1}\right| \leq 2 n-5$. Furthermore, $\left|F_{i}\right| \leq 2 n-5$ for $2 \leq i \leq n$. So $G_{i}-F_{i}$ has a fractional perfect matching $f_{i}$ for $1 \leq i \leq n$. Thus, $f_{1}, f_{2}, \cdots, f_{n}$ induce a fractional perfect matching of $S_{n}^{2}-F$.

If we can show that $S_{4}^{2}$ is fractional strongly super matched, then we can get our desired result from Theorem 6 that $S_{n}^{2}$ is fractional strongly super matched for $n \geq 4$. Fortunately, $S_{4}^{2}$ is fractional strongly super matched, which will be proved in Section 3. The following theorem is the main result of this paper.

Theorem 6 Let $n \geq 4$, then $f \operatorname{smp}\left(S_{n}^{2}\right)=2 n-3$. Moreover, $S_{n}^{2}$ is fractional strongly super matched.

\section{Initial Case}

We will show two initial cases. Let $G=\left(V_{G}, E_{G}\right)$ and $H=\left(V_{H}, E_{H}\right)$ be two graphs. Then their Cartesian product $G \square H$ is the graph with vertex set $V_{G} \square V_{H}=\left\{(u, v): u \in V_{G}, v \in V_{H}\right\}$, such that its vertices $(u, v)$ and $\left(u^{\prime}, v^{\prime}\right)$ are adjacent if and only if $u=u^{\prime}$ and $\left(v, v^{\prime}\right) \in E_{H}$, or $\left(u, u^{\prime}\right) \in E_{G}$ and $v=v^{\prime}$. In particular, $G \square K_{2}$ can be described as follows: Let $G_{1}$ and $G_{2}$ be two copies of $G$ such that $u \in V\left(G_{1}\right)$ and $u^{\prime} \in V\left(G_{2}\right)$ correspond to $u \in V(G)$. Then $G \square K_{2}$ is obtained by taking $G_{1}$ and $G_{2}$ with the edges of the form $\left(u, u^{\prime}\right)$ for every $u \in V(G)$. We call the edges of the form $\left(u, u^{\prime}\right)$ cross edges. Clearly $S_{n}^{2}=A_{n} \square K_{2}$. To prove the Theorem 6, we need to prove the Lemma 3 and Lemma 4. We start with the following Lemmas.

Lemma $3 f \operatorname{smp}\left(S_{4}^{2}\right)=5$.

Proof. Let $F \subseteq E\left(S_{4}^{2}\right) \cup V\left(S_{4}^{2}\right)$. Note that $S_{4}^{2}=A_{4} \square K_{2}$ is obtained by taking $G_{1}$ and $G_{2}$ with the edges of the form $\left(u, u^{\prime}\right)$, where $G_{i}$ is isomorphic to $A_{4}$ for $1 \leq i \leq 2, u \in V\left(G_{1}\right)$ and $u^{\prime} \in V\left(G_{2}\right)$. Let $F_{1}=F \cap G_{1}$ and $F_{2}=F \cap G_{2}$. Since $f \operatorname{smp}\left(S_{4}^{2}\right) \leq f m p\left(S_{4}^{2}\right)$ and $\operatorname{fmp}\left(S_{4}^{2}\right)=5$ by Theorem 4 , it follows that $f \operatorname{smp} p\left(S_{4}^{2}\right) \leq 5$. For notational convenience, assume $\left|F_{2}\right| \leq\left|F_{1}\right|$. Now we show the claim that $f \operatorname{smp}\left(S_{4}^{2}\right) \geq 5$, that is, for any $F \subseteq E\left(S_{4}^{2}\right) \cup V\left(S_{4}^{2}\right)$ with $|F| \leq 4, S_{4}^{2}-F$ has a fractional perfect matching. 
Case 1. $\left|F_{1}\right|=4$. Note that $\left|F_{2}\right|=0$. By Theorem $3, G_{1}-F_{1}$ satisfies one of the following: (1) $G_{1}-F_{1}$ has a perfect matching; (2) $G_{1}-F_{1}$ has an almost perfect matching; (3) $F_{1}$ is trivial SMP set and $x$ is an isolated vertex. As we saw above, $G_{1}-F_{1}$ has at most two vertices $x$ and $y$ that are unmatched. So $G_{1}-F_{1}-\{x, y\}$ has a fractional perfect matching $f_{1}$. Let $F_{2}^{\prime}=\left\{x^{\prime}, y^{\prime}\right\} \cap V\left(G_{2}\right)$, where $x^{\prime}$ and $y^{\prime}$ are neighbors of $x$ and $y$ in $G_{2}$, respectively. Since $\left|F_{2}^{\prime}\right|=2$, it follows that $G_{2}-F_{2}^{\prime}$ is Hamiltonian by Theorem 1 . So $G_{2}-F_{2}^{\prime}$ has a fractional perfect matching $f_{2}$. Thus $f_{1}$ and $f_{2}$ induce a fractional perfect matching of $S_{4}^{2}-F$.

Case 2. $\left|F_{1}\right|=3$. Note that $\left|F_{2}\right| \leq 1$ and $G_{1}$ is 4-regular. Since $\operatorname{smp}\left(A_{4}\right)=4$ by Theorem 3 , it follows that $G_{1}-F_{1}$ has either a perfect matching or an almost perfect matching. Assume that $G_{1}-F_{1}$ has a perfect matching $f_{1} . G_{2}-F_{2}$ is Hamiltonian by Theorem 1 , so $G_{2}-F_{2}$ has a fractional perfect matching $f_{2}$. Thus $f_{1}$ and $f_{2}$ induce a fractional perfect matching of $S_{4}^{2}-F$. We assume that $G_{1}-F_{1}$ has an almost perfect matching, that is, there exists a matching $M_{1}$ missing a vertex $u$ (If $F$ contains one cross edge of between $G_{1}$ and $G_{2}$, there exists a matching $M_{1}^{\prime}$ in $G_{1}-F_{1}$ missing a vertex $v$ such that $v$ is not incident with the cross edge in $F$ by Lemma 1.7). We would like to utilize the elements of $M_{1}$ to build fractional perfect matching in $G_{1}-F_{1}-\{u\}$. By Theorem $1, G_{2}-F_{2}-\left\{u^{\prime}\right\}$ is Hamiltonian, where $u^{\prime} \in V\left(G_{2}\right)$ and $u u^{\prime}$ is a cross edge, so $G_{2}-F_{2}-\left\{u^{\prime}\right\}$ has a fractional perfect matching $f_{2}$. These fact imply that vertex $u$ can be matched to vertex $u^{\prime}$ and then $M_{1} \cup\left\{\left(u u^{\prime}\right)\right\}$ and $f_{2}$ induce a fractional perfect matching of $S_{4}^{2}-F$.

Case 3. $\left|F_{1}\right| \leq 2$. Clearly, $\left|F_{2}\right| \leq 2$. Since $G_{1}-F_{1}$ and $G_{2}-F_{2}$ are Hamiltonian by Theorem 1 , it follows that $G_{1}-F_{1}$ and $G_{2}-F_{2}$ have fractional perfect matchings $f_{1}$ and $f_{2}$, respectively. Thus, $f_{1}$ and $f_{2}$ induce a fractional perfect matching of $S_{4}^{2}-F$.

A standard way to view $A_{4}$ is via its recursive structure. Let $H_{i}$ be the subgraph of $A_{4}$ induced by vertices where the last symbol is $i$, where $1 \leq i \leq 4$. Then $H_{i}$ is isomorphic to cycle of the three vertices. Each vertex $v$ in $H_{i}$ has exactly two neighbors outside of $H_{i}$; moreover, its two neighbors belong to different $H_{j}$ 's. We call these neighbors the externalneighbors of $v$. We call the edges whose end-vertices belong to different $H_{j}$ 's cross edges. Since the $H_{i}$ 's are defined via the 4th position, we say it is a decomposition via the 4th position. It is easy to see that for a given pair of $H_{i}$ and $H_{j}$, there are $(4-2) !=2$ ! cross edges between them; moreover, they are independent. We start with the following results.

\section{Lemma 4 Every optimal FSMP set of $S_{4}^{2}$ is trivial, that is, $S_{4}^{2}$ is fractional strongly super matched.}

Proof. Since $f \operatorname{smp}\left(S_{4}^{2}\right)=5$ by Lemma 3, it follows that we can complete the proof by showing that for any $F \subseteq$ $E\left(S_{4}^{2}\right) \cup V\left(S_{4}^{2}\right)$ with $|F|=5, S_{4}^{2}-F$ has a fractional perfect matching or $S_{4}^{2}-F$ has an isolated vertex such that $F$ is trivial FSMP set. So we only consider the case that $S_{4}^{2}-F$ has no isolated vertices. Note that $S_{4}^{2}=A_{4} \square K_{2}$. Let $F_{1}=F \cap G_{1}$ and $F_{2}=F \cap G_{2}$. Let $H_{1 i}$ be subgraph of $G_{1}$ induced by the set of vertices with $i$ in the last position for $1 \leq i \leq 4$. Let $F_{1 i}$ be the element of $F_{1}$ in $H_{1 i}$, where $1 \leq i \leq 4$. Let $H_{2 i}$ be subgraph of $G_{2}$ induced by the set of vertices with $i$ in the last position for $1 \leq i \leq 4$. Let $F_{2 i}$ be the element of $F_{2}$ in $H_{2 i}$, where $1 \leq i \leq 4$. For notational convenience, assume $\left|F_{2}\right| \leq\left|F_{1}\right|,\left|F_{1 i}\right| \leq\left|F_{11}\right|$ and $\left|F_{2 i}\right| \leq\left|F_{21}\right|$, where $2 \leq i \leq 4$. Now we show that $S_{4}^{2}-F$ has a fractional perfect matching.

Case 1. $\left|F_{1}\right|=5$. Let $F_{1}^{\prime}=F_{1}-\{\alpha\}$, where $\{\alpha\} \subseteq F_{1}$. By Theorem 3, $G_{1}-F_{1}^{\prime}$ satisfies one of the following: (1) $G_{1}-F_{1}^{\prime}$ has a perfect matching; (2) $G_{1}-F_{1}^{\prime}$ has an almost perfect matching; (3) $F_{1}^{\prime}$ is trivial SMP set, that is, $G_{1}-F_{1}^{\prime}$ has an isolated vertex $x$. We consider the following two possibilities according to $\alpha$.

Subcase 1.1. $\alpha$ is a vertex. Suppose $G_{1}-F_{1}^{\prime}$ has a perfect matching. So $G_{1}-F_{1}^{\prime}-\{\alpha\}$ has a vertex $\gamma$ that is unmatched, where $\gamma$ is a neighbor of $\alpha$ in $G_{1}$. This implies that $G_{1}-F_{1}^{\prime}-\{\alpha, \gamma\}$ has a fractional perfect matching $f_{1}$. Let $F_{2}^{\prime}=\left\{\gamma^{\prime}\right\} \cap V\left(G_{2}\right)$, where where $\gamma^{\prime}$ is a neighbor of $\gamma$ in $G_{2}$. Since $\left|F_{2}^{\prime}\right|=1$, it follows that $G_{2}-F_{2}^{\prime}$ is Hamiltonian by Theorem 1 , then $G_{2}-F_{2}^{\prime}$ has a fractional perfect matching $f_{2}$. Hence, $\left\{\left(\gamma \gamma^{\prime}\right)\right\}$ and $f_{1}, f_{2}$ induce a fractional perfect matching of $S_{4}^{2}-F$. Suppose $G_{1}-F_{1}^{\prime}$ has an almost perfect matching and a vertex $v$ that is unmatched. So $G_{1}-F_{1}^{\prime}-\{\alpha\}$ has two vertices $\gamma$ and $v$ that are unmatched, where $\gamma$ is a neighbor of $\alpha$ in $G_{2}$. This implies that $G_{1}-F_{1}^{\prime}-\{\alpha, \gamma, v\}$ has a fractional perfect matching $f_{1}$. Let $F_{2}^{\prime}=\left\{\gamma^{\prime}, v^{\prime}\right\} \cap V\left(G_{2}\right)$, where $\gamma^{\prime}$ is a neighbor of $\gamma$ in $G_{2}, v^{\prime}$ is a neighbor of $v$ in $G_{2}$. Since $\left|F_{2}^{\prime}\right|=2$, it follows that $G_{2}-F_{2}^{\prime}$ is Hamiltonian by Theorem 1 , then $G_{2}-F_{2}^{\prime}$ has a fractional perfect matching $f_{2}$. Hence, $\left\{\left(\gamma \gamma^{\prime}\right),\left(v v^{\prime}\right)\right\}$ and $f_{1}, f_{2}$ induce a fractional perfect matching of $S_{4}^{2}-F$. Suppose $F^{\prime}$ is trivial SMP set, that is, there are at most two vertices $x$ and $y$ in $G_{1}-F_{1}^{\prime}$ that are unmatched. So $G_{1}-F_{1}^{\prime}-\{\alpha\}$ has at most three vertices $\gamma, x$ and $y$ that are unmatched, where $\gamma$ is a neighbor of $\alpha$ in $G_{1}$. This implies that $G_{1}-F_{1}^{\prime}-\{\alpha, \gamma, x, y\}$ has a fractional perfect matching $f_{1}$. Let $F_{2}^{\prime}=\left\{\gamma^{\prime}, x^{\prime}, y^{\prime}\right\} \cap V\left(G_{2}\right)$, where $\gamma^{\prime}$ is a neighbor of $\gamma$ in $G_{2}, x^{\prime}$ is a neighbor of $x$ in $G_{2}$ and $y^{\prime}$ is a neighbor of $y$ in $G_{2}$. Note that $\left|F_{2}^{\prime}\right|=3$. If $F_{2}^{\prime}$ contains three vertices and $\left|F_{21}\right|=3, H_{2 i}-F_{2 i}$ has a fractional perfect matching $f_{2 i}$, where $2 \leq i \leq 4$. If $F_{2}^{\prime}$ contains three vertices, $\left|F_{21}\right|=2$ and $\left|F_{22}\right|=1$, then $H_{21}-F_{21}=\{v\}$ and $H_{22}-F_{22}$ is $K_{2}$. Since $v$ has two external-neighbors and $v$ is not an isolated vertex, there is an external-neighbor $v^{\prime}$ of $v$ in $H_{2 i}$, where $3 \leq i \leq 4$. Without loss of generality, assume $v^{\prime} \in V\left(H_{23}\right)$. It clear that $H_{22}-F_{22}$ and $H_{23}-\left\{v^{\prime}\right\}$ have perfect matchings $f_{22}$ and $f_{23}$, respectively, and $H_{24}$ has a fractional perfect matching $f_{24}$. If $F_{2}^{\prime}$ contains three vertices and $\left|F_{21}\right|=\left|F_{22}\right|=\left|F_{23}\right|=1$, then $H_{21}-F_{21}, H_{22}-F_{22}$ and $H_{23}-F_{23}$ are $K_{2}$, respectively. And $H_{24}$ has a fractional perfect matching $f_{24}$. So $G_{2}-F_{2}^{\prime}$ has a fractional perfect matching $f_{2}$. Hence, $\left\{\left(\gamma \gamma^{\prime}\right),\left(x x^{\prime}\right),\left(y y^{\prime}\right)\right\}$ and $f_{1}, f_{2}$ induce a fractional perfect matching of $S_{4}^{2}-F$. 
Subcase 1.2. $\alpha$ is an edge. Let $\alpha=u w$. Suppose $G_{1}-F_{1}^{\prime}$ has a perfect matching. So $G_{1}-F_{1}^{\prime}-\{\alpha\}$ has at most two vertices $u$ and $w$ that are unmatched. This implies that $G_{1}-F_{1}^{\prime}-\{\alpha, u, w\}$ has a fractional perfect matching $f_{1}$. Let $F_{2}^{\prime}=\left\{u^{\prime}, w^{\prime}\right\} \cap V\left(G_{2}\right)$, where $u^{\prime}$ is a neighbor of $u$ in $G_{2}$ and $w^{\prime}$ is a neighbor of $w$ in $G_{2}$. Since $\left|F_{2}^{\prime}\right|=2$, it follows that $G_{2}-F_{2}^{\prime}$ is Hamiltonian by Theorem 1 . So $G_{2}-F_{2}^{\prime}$ has a fractional perfect matching $f_{2}$. Therefore, $\left\{\left(u u^{\prime}\right),\left(w w^{\prime}\right)\right\}$ and $f_{1}, f_{2}$ induce a fractional perfect matching of $S_{4}^{2}-F$. Suppose $G_{1}-F_{1}^{\prime}$ has an almost perfect matching and a vertex $v$ that is unmatched. So $G_{1}-F_{1}^{\prime}-\{\alpha\}$ has at most three vertices $v, u$ and $w$ that are unmatched. This implies that $G_{1}-F_{1}^{\prime}-\{\alpha, v, u, w\}$ has a fractional perfect matching $f_{1}$. Let $F_{2}^{\prime}=\left\{v^{\prime}, u^{\prime}, w^{\prime}\right\} \cap V\left(G_{2}\right)$, where $v^{\prime}$ is a neighbor of $v$ in $G_{2}, u^{\prime}$ is a neighbor of $u$ in $G_{2}$ and $w^{\prime}$ is a neighbor of $w$ in $G_{2}$. Since $\left|F_{2}^{\prime}\right|=3$, it follows that $G_{2}-F_{2}^{\prime}$ has a fractional perfect matching $f_{2}$. Hence, $\left\{\left(u u^{\prime}\right),\left(w w^{\prime}\right),\left(v v^{\prime}\right)\right\}$ and $f_{1}, f_{2}$ induce a fractional perfect matching of $S_{4}^{2}-F$. Suppose $F_{1}$ is trivial SMP set, that is, there are at most two vertices $x$ and $y$ in $G_{1}-F_{1}^{\prime}$ that are unmatched. So $G_{1}-F_{1}^{\prime}-\{\alpha\}$ has at most four vertices $x, y, u$ and $w$ that are unmatched. This implies that $G_{1}-F_{1}^{\prime}-\{\alpha, x, y, u, w\}$ has a fractional perfect matching $f_{1}$. Let $F_{2}^{\prime}=\left\{x^{\prime}, y^{\prime}, u^{\prime}, w^{\prime}\right\} \cap V\left(G_{2}\right)$, where $x^{\prime}$ is a neighbor of $x$ in $G_{2}, y^{\prime}$ is a neighbor of $y$ in $G_{2}, u^{\prime}$ is a neighbor of $u$ in $G_{2}$, and $w^{\prime}$ is a neighbor of $w$ in $G_{2}$. Note that $\left|F_{2}^{\prime}\right|=4$ and $H_{2 i}$ is isomorphic to a cycle of three vertices, where $1 \leq i \leq 4$. If $\left|F_{21}\right|=3$ and $\left|F_{22}\right|=1$, then $H_{22}-F_{22}$ is $K_{2}$. So $H_{22}-F_{22}$ has a fractional perfect matching $f_{22}$. Clearly, $H_{2 i}-F_{2 i}$ has fractional perfect matching $f_{2 i}$, where $3 \leq i \leq 4$. So $f_{22}, f_{23}$ and $f_{24}$ induce a fractional perfect matching of $G_{2}-F_{2}^{\prime}$. Thus, $f_{1}, f_{22}, f_{23}$ and $f_{24}$ induce a fractional perfect matching of $S_{4}^{2}-F$. If $\left|F_{21}\right|=2$ and $\left|F_{22}\right|=2$, then $H_{21}-F_{21}$ is an isolated vertex $x$ and $H_{22}-F_{22}$ is an isolated vertex $y$. We may select an external-neighbor for each vertex from $\{x, y\}$ such that they are in different $H_{2 i}$ 's, where $3 \leq i \leq 4$, otherwise, we can decompose $G_{2}$ by choosing a new position. Assume $x^{\prime} \in V\left(H_{23}\right)$ and $y^{\prime} \in V\left(H_{24}\right)$. Then $H_{23}-\left\{x^{\prime}\right\}$ and $H_{24}-\left\{y^{\prime}\right\}$ are two $K_{2}$. So $H_{23}-\left\{x^{\prime}\right\}$ and $H_{24}-\left\{y^{\prime}\right\}$ have fractional perfect matching $f_{23}$ and $f_{24}$. Thus $f_{1}, f_{23}$ and $f_{24}$ induce a fractional perfect matching of $S_{4}^{2}-F$. If $\left|F_{21}\right|=2,\left|F_{22}\right|=1$ and $\left|F_{23}\right|=1$, then $H_{21}-F_{21}$ has an isolated vertex $x, H_{22}-F_{22}$ and $H_{23}-F_{23}$ are two $K_{2}$. We may select an external-neighbor of $x$ in $H_{24}$, say $x^{\prime} \in V\left(H_{24}\right)$, otherwise, we can decompose $G_{2}$ by choosing a new position. Then $H_{22}-F_{22}$ and $H_{23}-F_{23}$ has a fractional perfect matching $f_{2 i}$, where $2 \leq i \leq 3$. And $H_{24}-\left\{x^{\prime}\right\}$ has a fractional perfect matching $f_{24}$. Therefore, $f_{1}, f_{22}, f_{23}$ and $f_{24}$ induce a fractional perfect matching of $S_{4}^{2}-F$. If $\left|F_{21}\right|=\left|F_{22}\right|=\left|F_{23}\right|=\left|F_{24}\right|=1$, then $H_{2 i}-F_{2 i}$ is $K_{2}$, where $1 \leq i \leq 4$. So $f_{21}, f_{22}, f_{23}$ and $f_{24}$ induce a fractional perfect matching of $G_{2}-F_{2}^{\prime}$. Therefore, $f_{1}, f_{21}, f_{22}, f_{23}$ and $f_{24}$ induce a fractional perfect matching of $S_{4}^{2}-F$.

Case 2. $\left|F_{1}\right|=4$. Clearly, $\left|F_{2}\right| \leq 1$. By Theorem 3, $G_{1}-F_{1}$ satisfies one of the following: (1) $G_{1}-F_{1}$ has a perfect matching; (2) $G_{1}-F_{1}$ has an almost perfect matching; (3) $F_{1}$ is trivial SMP set and $x$ is an isolated vertex. Suppose $G_{1}-F_{1}$ has a perfect matching, that is, $G_{1}-F_{1}$ has a fractional perfect matching $f_{1}$. Since $\left|F_{2}\right| \leq 1$, it follows that $G_{2}-F_{2}$ is Hamiltonian by Theorem 1 , then $G_{2}-F_{2}$ has a fractional perfect matching $f_{2}$. Therefore, $f_{1}$ and $f_{2}$ induce a fractional perfect matching of $S_{4}^{2}-F$. Suppose $G_{1}-F_{1}$ has an almost perfect matching, that is, $G_{1}-F_{1}$ has a vertex $v$ that is unmatched. So $G_{1}-F_{1}-\{v\}$ has a fractional perfect matching $f_{1}$ by Lemma 1 . Let $F_{2}^{\prime}=\left(\left\{v^{\prime}\right\} \cap V\left(G_{2}\right)\right) \cup F_{2}$, where $v^{\prime}$ is a neighbor of $v$ in $G_{2}$. Since $\left|F_{2}\right| \leq 1$, clearly, $\left|F_{2}^{\prime}\right| \leq 2$. So $G_{2}-F_{2}^{\prime}$ is Hamiltonian by Theorem 1, then $G_{2}-F_{2}$ has a fractional perfect matching $f_{2}$. Thus $f_{1}$ and $f_{2}$ induce a fractional perfect matching of $S_{4}^{2}-F$. Suppose $F_{1}$ is trivial SMP set and $x$ is an isolated vertex, that is, $G_{1}-F_{1}$ has at most two vertices $x$ and $y$ that are unmatched. Let $F_{2}^{\prime}=\left(\left\{x^{\prime}, y^{\prime}\right\} \cap V\left(G_{2}\right)\right) \cup F_{2}$, where $x^{\prime}$ is a neighbor of $x$ in $G_{2}, y^{\prime}$ is a neighbor of $y$ in $G_{2}$. Since $\left|F_{2}^{\prime}\right| \leq 3 . G_{2}-F_{2}^{\prime}$ has a fractional perfect matching $f_{2}$ by Case 1 . Thus $f_{1}$ and $f_{2}$ induce a fractional perfect matching of $S_{4}^{2}-F$.

Case 3. $\left|F_{1}\right|=3$ and $\left|F_{2}\right| \leq 2$. Since $G_{2}-F_{2}$ is Hamiltonian by Theorem 1, it follows that $G_{2}-F_{2}$ has a fractional perfect matching $f_{2}$. It follows from Theorem 3 that we only consider the case that $F_{1}$ consists of an odd number of vertices. As we have now seen, if $F_{1}$ contains three vertices and $\left|F_{11}\right|=3$, then $H_{1 i}-F_{1 i}$ has a fractional perfect matching $f_{1 i}$, where $2 \leq i \leq 4$. So $f_{12}, f_{13}, f_{14}$ and $f_{2}$ induce a fractional perfect matching of $S_{4}^{2}-F$. If $F_{1}$ contains three vertices, $\left|F_{11}\right|=2$ and $\left|F_{12}\right|=1$, then $H_{11}-F_{11}=\{v\}$, and $H_{12}-F_{12}$ is $K_{2}$. Since $v$ has two external-neighbors and $v$ is not an isolated vertex, there is an external-neighbor $v^{\prime}$ of $v$ in $H_{1 i}$, where $3 \leq i \leq 4$. Without loss of generality, assume $v^{\prime} \in V\left(H_{13}\right)$. It clear that $H_{12}-F_{12}$ and $H_{13}-\left\{v^{\prime}\right\}$ have perfect matchings $f_{12}$ and $f_{13}$, respectively, and $H_{14}$ has a fractional perfect matching $f_{4}$. So $\left\{\left(v v^{\prime}\right)\right\}$ and $f_{12}, f_{13}, f_{14}, f_{2}$ induce a fractional perfect matching of $S_{4}^{2}-F$. If $F_{1}$ contains three vertices and $\left|F_{11}\right|=\left|F_{12}\right|=\left|F_{13}\right|=1$, then $H_{11}-F_{11}, H_{12}-F_{12}$ and $H_{13}-F_{13}$ are $K_{2}$, respectively. $H_{1 i}-F_{1 i}$ has fractional perfect matching $f_{1 i}$, where $1 \leq i \leq 3$. And $H_{14}$ has a fractional perfect matching $f_{14}$, so $f_{11}, f_{12}, f_{13}, f_{14}, f_{2}$ induce a fractional perfect matching of $S_{4}^{2}-F$. Next we consider the case that $F_{1}$ contains one vertex and two edges. If $F_{11}$ consists of one vertex and two edges, $H_{11}-F_{11}$ has at most two isolated vertices, say $u$ and $v$. We may select an external-neighbor for each vertex from $\{u, v\}$ such that they are in different $H_{1 i}$ 's, where $2 \leq i \leq 4$. For notational convenience, assume $u^{\prime} \in V\left(H_{12}\right)$ and $v^{\prime} \in V\left(H_{13}\right)$, where $u^{\prime}$ is an external-neighbor of $u$ in $V\left(H_{12}\right), v^{\prime}$ is an external-neighbor of $v$ in $V\left(H_{13}\right)$. So $H_{12}-\left\{u^{\prime}\right\}$ and $H_{13}-\left\{v^{\prime}\right\}$ have fractional perfect matchings $f_{12}$ and $f_{13}$, respectively. $H_{14}$ has a fractional perfect matching $f_{14}$. So $f_{12}, f_{13}, f_{14}$ and $f_{2}$ induce a fractional perfect matching of $S_{4}^{2}-F$. If $F_{11}$ consists of one vertex and one edge, and $F_{12}$ contains one edge. $H_{11}-F_{11}$ has at most two isolated vertices, say $u$ and $v$, and $H_{12}-F_{12}$ is a path $P$ with three vertices. Let $P=x y z$. We can find that the external-neighbor of one of $u$ and $v$ is adjacent to one of $x$ and $z$, otherwise, we can decompose $G_{1}$ by choosing a new position. Without loss of generality, assume that $u$ is adjacent to $x$. Note that 
there is the external-neighbor $v^{\prime}$ of $v$ in $H_{1 i}$, where $3 \leq i \leq 4$. Assume $v^{\prime} \in V\left(H_{13}\right)$, then $H_{13}-\left\{v^{\prime}\right\}$ has a fractional perfect matching $f_{13}$. Clearly, $H_{14}$ has a fractional perfect matching $f_{14}$. So $\left\{(u x),(y z),\left(v v^{\prime}\right)\right\}$ and $f_{13}, f_{14}, f_{2}$ induce a fractional perfect matching of $S_{4}^{2}-F$. If $F_{1}$ contains cross edges such that $F_{11}$ consists of one vertex and $F_{12}$ contains one edge, we can obtain $H_{11}-F_{11}$ has a fractional perfect matching $f_{11}$ and $H_{12}-F_{12}$ is a path $P=u v w$ with three vertices. It obvious that we can find the external-neighbor $u^{\prime}$ of $u$ in $H_{1 i}$, where $3 \leq i \leq 4$. Assume $u^{\prime} \in V\left(H_{13}\right)$. Moreover, $H_{13}-\left\{u^{\prime}\right\}$ and $H_{14}$ have fractional perfect matchings $f_{13}$ and $f_{14}$, respectively. When $F_{1}$ contains no cross edges, we can choose a new position to decompose $G_{1}$ such that $\left|F_{12} \cap E\left(H_{12}\right)\right|=1$ and $F_{11}$ consists of one vertex. So $\left\{\left(u u^{\prime}\right),(v w)\right\}$ and $f_{11}, f_{13}, f_{14}$ induce a fractional perfect matching of $G_{1}-F_{1}$. Thus $\left\{\left(u u^{\prime}\right),(v w)\right\}$ and $f_{11}, f_{13}, f_{14}, f_{2}$ induce a fractional perfect matching of $S_{4}^{2}-F$.

Case 4. $\left|F_{1}\right| \leq 2$. By the Case 2 and Case $3, S_{4}^{2}-F$ has a fractional perfect matching.

Thus, we prove that every optimal FSMP set of $S_{4}^{2}$ is trivial, that is, $S_{4}^{2}$ is fractional strongly super matched.

With Lemma 3 and Lemma 4 proved, we immediately obtain the following result.

Theorem $7 f \operatorname{smp}\left(S_{4}^{2}\right)=5$. Moreover, $S_{4}^{2}$ is fractional strongly super matched.

\section{References}

Birgham, R. C., Harry, F., Biolin, E. C., \& Yellen, J. (2005), Perfect matching preclusion, Congr. Numer, 174, 185-192.

Bonneville, P., Cheng, E., \& Renzi, J. (2011). Strong matching preclusion for the alternating groups and split-stars, JOIN 4, 277-298.

Cheng, E., Lesniak, L., Lipman, M. J., \& Lipták, L. (2008). Matching preclusion for alternating group graphs and their generalizations. Inter. J. Found. Comput. Sci. 19, 1413-1437.

Cheng, E., Lesniak, L., Lipman, M. J., \& Lipták, L. (2009). Conditional matching preclusion sets, Inform. Sci. 179, 1092-1101.

Cheng, E., Lipman, M. J., \& Park, H. A. (2001). Super connectivity of star graphs, alternating group graphs and split-stars, Ars Combin. 59, 107-116.

Cheng, E., \& Lipták, L. (2007). Matching preclusion for some interconnection networks, Networks, 50, 173-180.

Cheng, E., \& Siddiqui, O. (2016). Strong matching preclusion of arrangement graphs, JOIN 16, 1650004.

Day, K., \& Tripathi, A. (1992). Arrangement graphs: a class of generalized star graphs. Inf. Process. Lett, 42, 235-241.

Hsu, H. C., Li, T. K., Tan, J. J. M., \& Hsu, L. H. (2004). Fault Hamiltonicity and fault Hamiltonian connectivity of the arrangement graphs, IEEE Transactions on Computers, 53, 39C53.

Jwo, J. S., Lakshmivarahan, S., \& Dhall, S. K. (1993). A new class of interconnection networks based on the alternating group, Networks 23, 315-326.

Lin, R., \& Zhang, H. (2017). Matching preclusion and conditional edge-fault Hamiltonicity of binary de Bruijn graphs, Discrete Appl. Math. 233, 104-117.

Liu, Y., \& Liu, W. (2016). Fractional matching preclusion number of graphs, J. Comb. Optim. 34, 522-533.

Ma, T., Mao, Y., Cheng, E., \& Melekian, C. (2018). Fractional matching preclusion for (burnt) pancake graphs, I-SPAN. 00030, 133-141.

Ma, T., Mao, Y., Cheng, E., \& Wang, J. (2018). Fractional matching preclusion for $(n, k)$-star graphs, Parall. Process. Lett. 28, 1850017.

Mao, Y., Wang, Z., Cheng, E., \& Melekian, C. (2018). Strong matching preclusion number of graphs, Theor. Comput. Sci. 713, 11-20.

Park, J. H., \& Ihm, I. (2011). Strong Matching Preclusion,Theor. Comput. Sci. 412, 6409-6419.

Scheinerman, E. R., \& Ullman, D. H. (1997) Fractional Graph Theory: A Rational Approach to the Theory of Graphs, John Wiley, New York.

Wang, Z., Mao, Y., Cheng, E., \& Zou, J. (2019). Matching preclusion number of graphs, Theor. Comput. Sci. 759, 61-71.

Wang, Z., Melekian, C., Cheng, E., \& Mao, Y. (2019). Matching preclusion number in product graphs, Theor. Comput. Sci. 755, 38-47. 


\section{Copyrights}

Copyright for this article is retained by the author(s), with first publication rights granted to the journal.

This is an open-access article distributed under the terms and conditions of the Creative Commons Attribution license (http://creativecommons.org/licenses/by/4.0/). 